\title{
Dr. Reggie J. Laird Broom: pionero de la ciencia del suelo en México
}

\section{Dr. Reggie J. Laird Broom: pioneer of soil science in Mexico}

\section{David Espinosa Victoria ${ }^{1 *}$}

\footnotetext{
${ }^{1}$ Colegio de Postgraduados, Programa de Postgrado en Edafología. Carretera México-Texcoco km. 36.5. 56230 Montecillo, Estado de México, México. *Autor responsable (despinos@colpos.mx)
}

\section{RESUMEN}

A casi tres años de su deceso, la Sociedad Mexicana de la Ciencia del Suelo, A.C. rinde, a través de esta reseña, un homenaje póstumo a la memoria de uno de los pioneros de la Ciencia del Suelo en México. El trabajo realizado por el Dr. Reggie J. Laird Broom (Figura 1), a lo largo de 45 años, refleja de forma contundente su compromiso y pasión por ayudar a los pequeños productores de México, América Latina y el mundo.

\section{SUMMARY}

Nearly three years after his death, the Mexican Society of Soil Science, A.C., through this review, pays posthumous tribute to the memory of one of the pioneers of Soil Science in Mexico. The work done by Dr. Reggie J. Laird Broom (Figure 1) over 45 years emphatically reflects his commitment to and passion for helping small farmers in Mexico, Latin America and the world.

Figura 1. Dr. Reggie J. Laird Broom

(1920-2014)

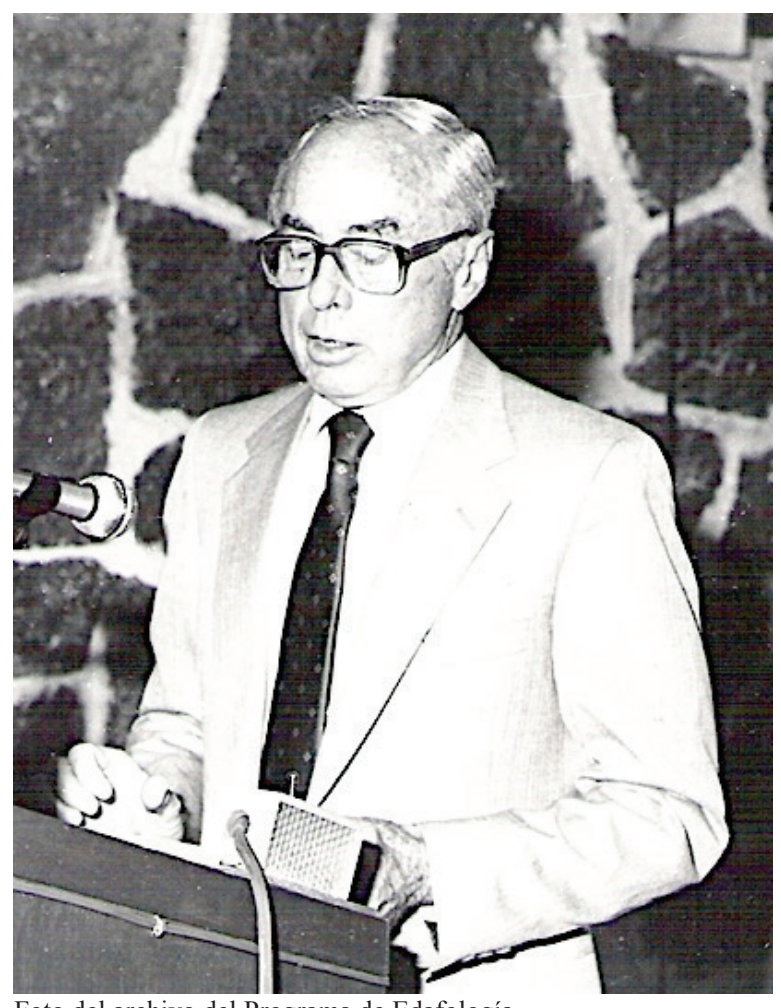

Foto del archivo del Programa de Edafología

Cita recomendada:

Espinosa Victoria, D. 2017. Semblanza del Dr. Reggie J. Laird Broom: pionero de la ciencia del suelo en México. Terra Latinoamericana 35: 1-6.
Recibido: septiembre de 2016. Aceptado: noviembre de 2016. Publicado en Terra Latinoamericana 35: 1-6. 


\section{Origen y Formación Académica}

El Dr. Reggie J. Laird Broom nació el 11 de febrero de 1920 en Bassfield, Mississippi, USA. Creció en un Mississippi rural, durante la época de la gran depresión norteamericana (1929-1932). El Dr. Laird fue uno de nueve hijos de una familia campesina, dedicada a la pizca del algodón. Aunque su familia nunca pasó hambre, el exceso de alimento no fue común en ésta.

Después de graduarse en la escuela media superior a la edad de 16 años, ingresó a la Universidad Estatal de Mississippi para cursar la carrera de Ingeniería. No obstante, la nostalgia cambio su objetivo y optó por estudiar Fertilidad de Suelos, pensando que era su deber permanecer en la finca de la familia. Fue en 1940 cuando obtuvo su grado de Bachelor of Science.

Más tarde, el Dr. Laird ingresa a la Universidad de Wisconsin-Madison, para cursar su maestría y doctorado. Recibió el grado de Maestro en Ciencias en 1942, justo cuando los Estados Unidos ingresaron a la Segunda Guerra Mundial. Por esta razón, el Dr. Laird dejó sus estudios para servir al gobierno norteamericano en la Guerra del Pacífico (Pacific Theater). A su regreso a la Unión Americana, el Dr. Laird completa sus estudios doctorales en 1952 en la Universidad de California-Berkeley (Heidbrink, 2014).

En 1945, el Dr. Laird fue condecorado por la Armada de los Estados Unidos con la Estrella de Plata, por su notable gallardía e intrepidez en la acción contra el enemigo mientras sirvió en la División 33 de infantería durante la Segunda Guerra Mundial.

\section{Su Llegada a México}

El Dr. Laird recibió a finales de 1951 una invitación de la Fundación Rockeffeller para visitar México y conocer el programa de suelos de la Oficina de Estudios Especiales, dependiente de la Secretaría de Agricultura, creada en 1943 a través de un convenio entre el Gobierno Mexicano y la Fundación Rockefeller. El Dr. Laird visitó México por primera vez en 1952, prácticamente al término de sus estudios doctorales, para una entrevista de trabajo con dicha Fundación. La tarea era contribuir a la mejora de la productividad de los suelos agrícolas. Sin embargo, la pobreza que observó en el país fue tal que, se desanimó hasta el punto de rechazar la oferta de trabajo. Así, retornó a la universidad, pero su profesor asesor lo invitó a reflexionar y tomar una decisión más fundamentada.
Afortunadamente, el Dr. Laird cambió su decisión y regresó a México para realizar investigación en la Ciencia del Suelo. El Dr. Pitner, jefe del Departamento de Suelos de la Oficina de Estudios Especiales, le encargó la generación de recomendaciones sobre el uso de fertilizantes, principalmente en maíz, trigo, frijol y papa bajo condiciones de riego y temporal. Las estaciones experimentales estratégicas en las que llevó a cabo sus investigaciones fueron Ciudad Obregón en el noroeste, La Cal Grande en el Bajío, El Horno en el Altiplano y Cotaxlta en el Trópico Húmedo.

Sin duda, con su arduo trabajo, el Dr. Laird ayudó a alimentar al mundo. Su esposa, la Sra. Rosario Laird dijo en una entrevista: "Él se dio cuenta que eso es lo que quería hacer. Él quería hacer la diferencia y la hizo. Fue el mejor ser humano que jamás pude haber conocido" (Heidbrink, 2014).

En resumen, el Dr. Laird desarrolló tecnología para el uso eficiente de los fertilizantes con diferentes prácticas de producción tales como la densidad de población, fechas de siembra y uso del agua de riego, entre otras. El uso de esta tecnología formó parte de lo que se conoció como "El Milagro Mexicano" ocurrido en las décadas de 1950 y 1960. Sin lugar a dudas, como lo indica el Dr. Antonio Turrent: "Reggie J. Laird es el Padre de la Fertilidad de Suelos en México" (Jiménez et al., 2014).

\section{Partícipe y Fundador de Instituciones Agrícolas en México}

Dada la naturaleza de la actividad agrícola, el Dr. Laird estuvo, desde su llegada a México, estrechamente relacionado con la Escuela Nacional de Agricultura (hoy Universidad Autónoma Chapingo) y su Colegio de Postgraduados, fundado en 1959.

El Dr. Laird participó en el Colegio de Postgraduados desde su creación en actividades de enseñanza e investigación. En 1959, el cuerpo de profesores de la Rama de Suelos estaba integrado por el MSc. Nicolás Aguilera Herrera en Pedología, el M.C. Mariano Villegas Soto en Física de Suelos, el Dr. Ramón Fernández González en Relaciones AguaSuelo-Planta-Atmósfera, el Dr. Enrique Ortega Torres en Química de Suelos y el Dr. Reggie J. Laird en Tecnología de Fertilizantes.

A partir de 1974, año en el cual la operación del Plan Puebla (ver más adelante) quedó bajo la responsabilidad del Colegio de Postgraduados, 
el Dr. Laird pasó definitivamente a formar parte de su personal académico. En 1976 recibió el nombramiento de Profesor Investigador Titular en la otrora Rama de Suelos, hoy Programa de Postgrado en Edafología del Campus Montecillo.

Inicialmente, el Dr. Laird impartió el curso de "Tecnología de Fertilizantes" (EDA-633) y más tarde el de "Fertilidad de Suelos" (EDA-631). De 1983 a 1995, impartió el curso "Investigación Agronómica para el Desarrollo de la Agricultura Tradicional" (EDA-661), diseñado por el mismo, así como el texto empleado.

Participó como Profesor Consejero y Asesor de ocho y 37 estudiantes, respectivamente. Del primer grupo, uno fue de doctorado y los otros siete de maestría. De los estudiantes asesorados, nueve fueron de doctorado y 28 de maestría (Jiménez et al., 2014).

Sus investigaciones sobre la fertilidad del suelo le permitieron conocer las principales regiones agrícolas de México, así como sus diversos sistemas agrícolas. Estos conocimientos lo habilitaron para llevar a cabo investigaciones en parcelas de agricultores en el Bajío, la Meseta Purépecha en Michoacán y otras regiones agrícolas del país.

Comprometido con el gremio de los estudiosos del suelo, Dr. Laird plasmó su nombre y firma en el Acta Constitutiva de la Sociedad Mexicana de la Ciencia del Suelo (SMCS) A.C., fundada el 19 de octubre de 1962 (Palacios, 2011).
En 1960, al crearse el Instituto de Investigaciones Agrícolas (IIA), ingresó como parte de su personal de investigación. De la misma forma, en 1966, participó como investigador fundador del Centro Internacional de Mejoramiento de Maíz y Trigo (CIMMYT).

En 1989, el Dr. Laird honró a la comunidad edafológica del Colegio de Postgraduados al plantar un ahuehuete (Figura 2) con motivo de la celebración del XXX aniversario de la Especialidad de Edafología.

\section{Su Participación en el Plan Puebla}

Como miembro del CIMMYT y del Colegio de Postgraduados, en 1967 participó en el diseño del Proyecto Puebla. Durante este período, el Dr. Laird llevó a cabo investigación científica en parcelas de los agricultores, con el objeto de generar tecnología apropiada para la producción de maíz a pequeña escala bajo condiciones de agricultura de temporal en el área del Proyecto Puebla, y más tarde en regiones agrícolas de ocho estados de la República Mexicana.

El Dr. Laird, conocedor profundo de las circunstancias de los pequeños agricultores, estimuló el trabajo de investigación para incrementar la productividad de la mano de obra familiar, mediante el desarrollo de nuevos implementos agrícolas de tracción animal, como la sembradora-fertilizadora línea Plan Puebla (Figura 3) (Jiménez et al., 2014).

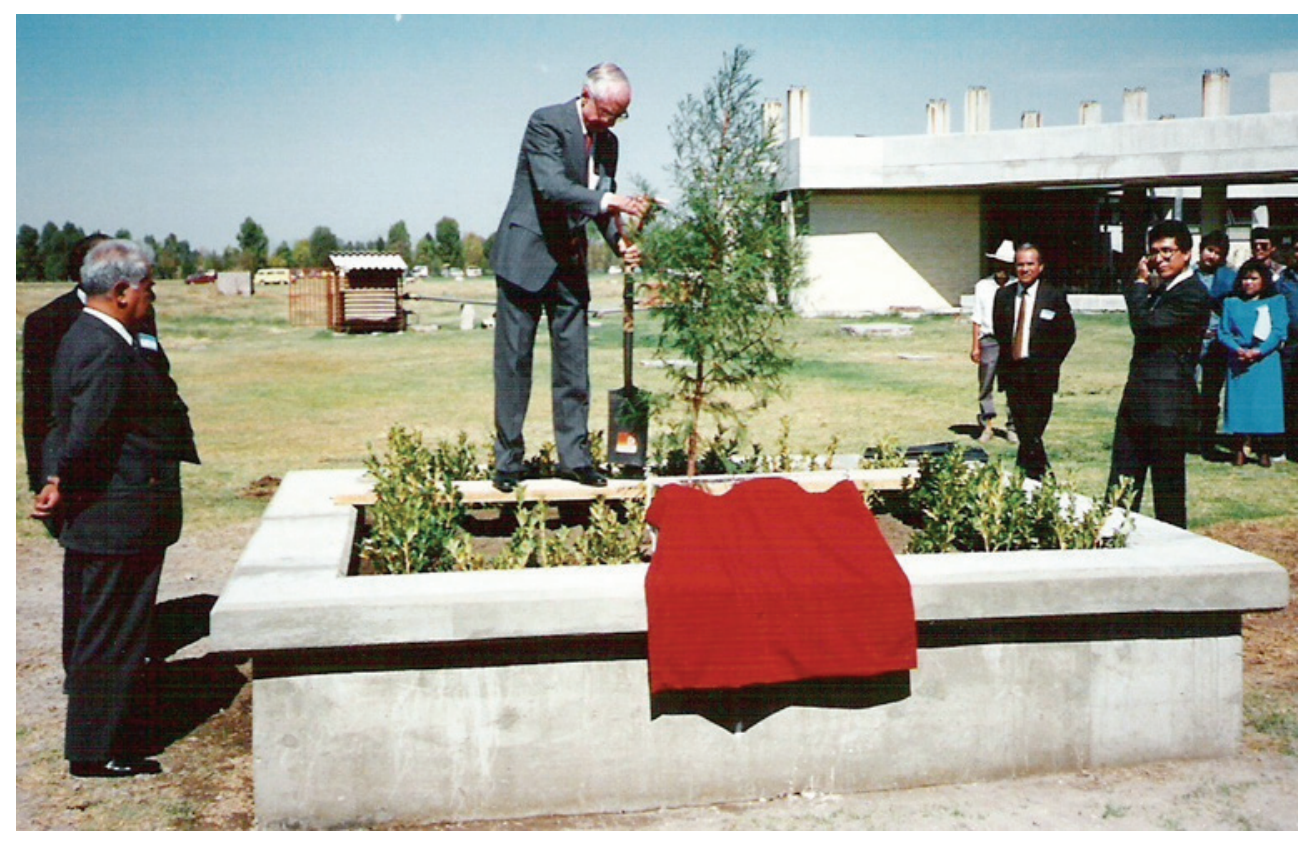

Figura 2. En 1989 se conmemoró el XXX Aniversario de la Especialidad de Edafología, el Dr. Reggie J. Laird Broom plantó un ahuehuete (foto del archivo del Programa de Edafología). 


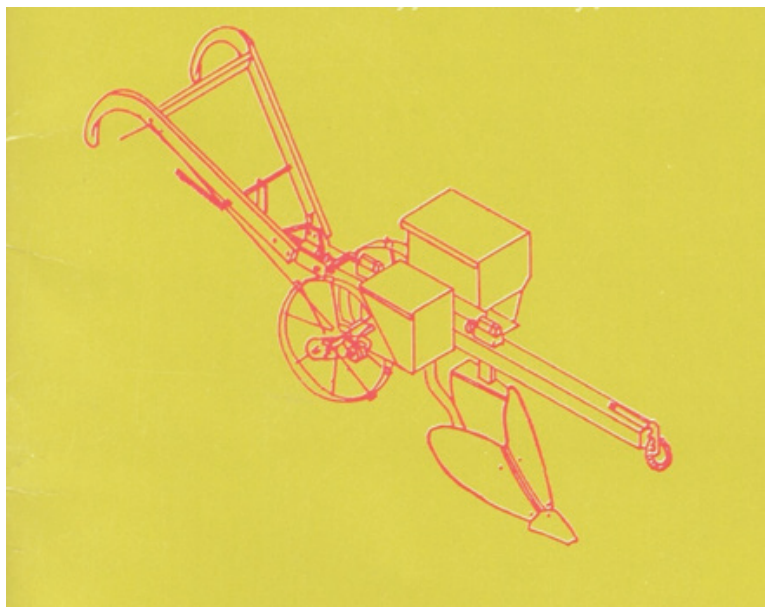

Figura 3. Prototipo de sembradora-fertilizadora diseñado en el Plan Puebla (Mendoza et al., 1994).

Captaron su atención los diferentes sistemas de producción diseñados por los mismos productores, particularmente la asociación de árboles frutales con maíz y frijol (Jiménez et al., 2014). De hecho, el sistema Milpa Intercalada con Árboles Frutales (MIAF) es resultado de las lecciones aprendidas en el Plan Puebla, en el que el Dr. Laird jugó un papel crucial.

La vida del Dr. Laird fue de intenso trabajo, tanto en el aula como en el campo. El Plan Puebla fue el marco idóneo para interactuar con un sinnúmero de investigadores, entre los que se encontraban algunos de sus otrora estudiantes como Armando Puente Berumen, Antonio Turrent Fernández, Álvaro Ruiz Barbosa, Benjamín Peña Olvera, Claudio Esquivel Sánchez, Alfonso Macías Laylle, Nestor Estrella Chulín y José Isabel Cortés, entre otros (Colpos, 1999).

Con toda certeza, el Dr. Cortes apunta que "mientras existan pequeños agricultores, sus enseñanzas de cómo investigar y enseñar sobre el desarrollo de tecnologías alternativas sustentables, serán siempre vigentes" (Jiménez et al., 2014). En la Figura 4, se muestra una panorámica de la última visita de campo que realizó el Dr. Laird en 2013 a los trabajos de investigación con la escuela del Plan Puebla en la Región Mixe, Oaxaca. Esta foto es muy significativa, porque además de ser la última visita al campo, muestra su cercanía con los pequeños productores y con sus colegas y colaboradores.

\section{Su Proyección de México al Mundo}

Como resultado de las experiencias derivadas del Plan Puebla, a partir de 1980 desarrolló actividades de capacitación de personal técnico y asesoría en investigación sobre productividad de agrosistemas en países de África, Asia y América Latina, a través del convenio firmado entre el Colegio de Postgraduados y el Comité Internacional de Enlace de Programas para la Alimentación (CILCA).

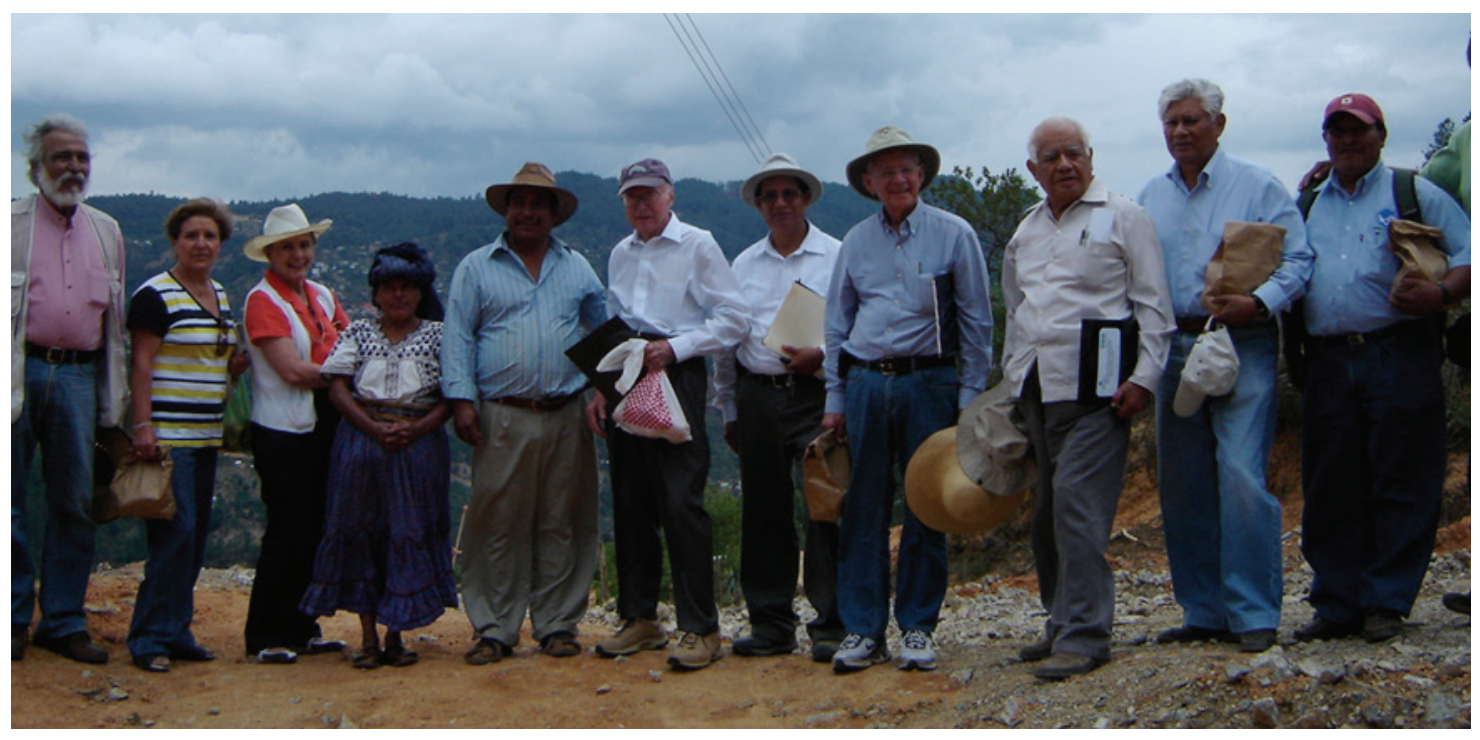

Figura 4. Última visita de campo que el Dr. Laird realizó en 2013 a los trabajos del Plan Puebla en la Región Mixe, Oaxaca. De izquierda a derecha: Heliodoro Díaz Cisneros, Magdalena Martínez Escobar, Rosario Shroeder de Laird (Chayito), Julia González de Jiménez, Filemón Jiménez Pacheco (productor del predio), Reggie J. Laird, José I. Cortes Flores, Antonio Turrent Fernández, Leobardo Jiménez Sánchez, Ángel Ramos Sánchez y Odilón Martínez Martínez (foto tomada de Jiménez et al., 2014). 


\section{Reconocimientos}

La solidez científica del Dr. Laird fue reconocida por el Sistema Nacional de Investigadores (SNI) desde 1984, año de su creación. En 1993 se le dio el nombramiento de Investigador Nacional Emérito, la máxima categoría otorgada por el SNI. Fue autor de más de 75 publicaciones nacionales e internacionales, y coadyuvó a la escritura de los informes de 25 años de investigación, operación y evaluación del Plan Puebla.

En 1999, por acuerdo del Consejo Técnico, el Colegio de Postgraduados otorgó al Dr. Laird el Doctorado Honoris causa (Figura 5) por el arduo trabajo realizado en favor de los que menos tienen, no solo de México, sino también de América Latina y África.

En el marco del Cincuentenario de la SMCS, AC, celebrado en el XXXVII Congreso Nacional en Zacatecas, Zac. en 2012, se le entregó la placa que lo reconoce como Miembro Fundador de la misma (Figura 6). Dado su estado de salud, el Dr. Laird no asistió a la ceremonia; no obstante, el reconocimiento le fue enviado a San Antonio, Texas, USA, gesto que su familia agradeció con una efusiva carta.

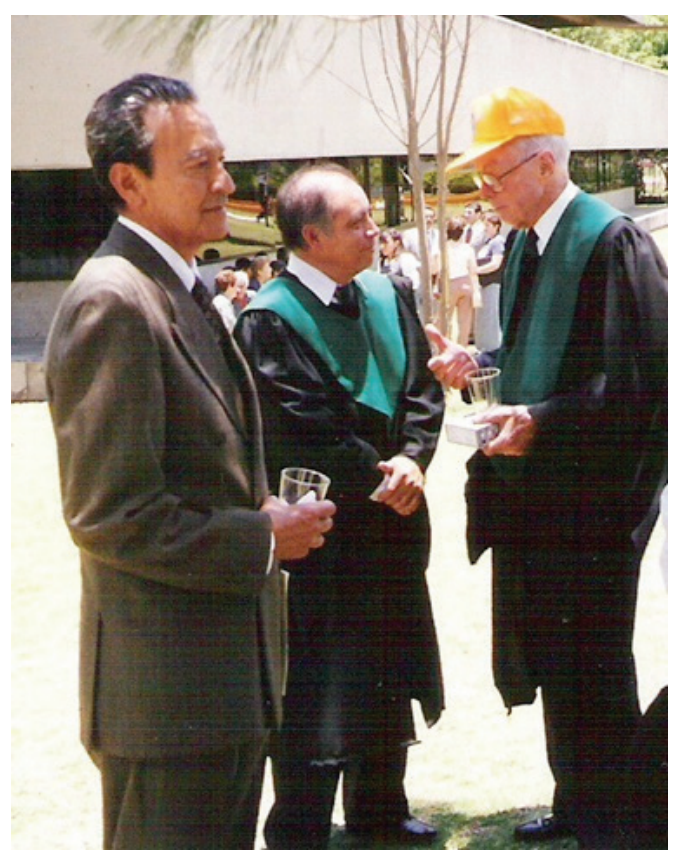

Figura 5. En 1999 el Colegio de Postgraduados otorgó al Dr. Laird Broom el Doctorado Honoris causa. En la foto aparece con el Dr. Roberto Núñez Escobar (foto del archivo del Programa de Edafología).

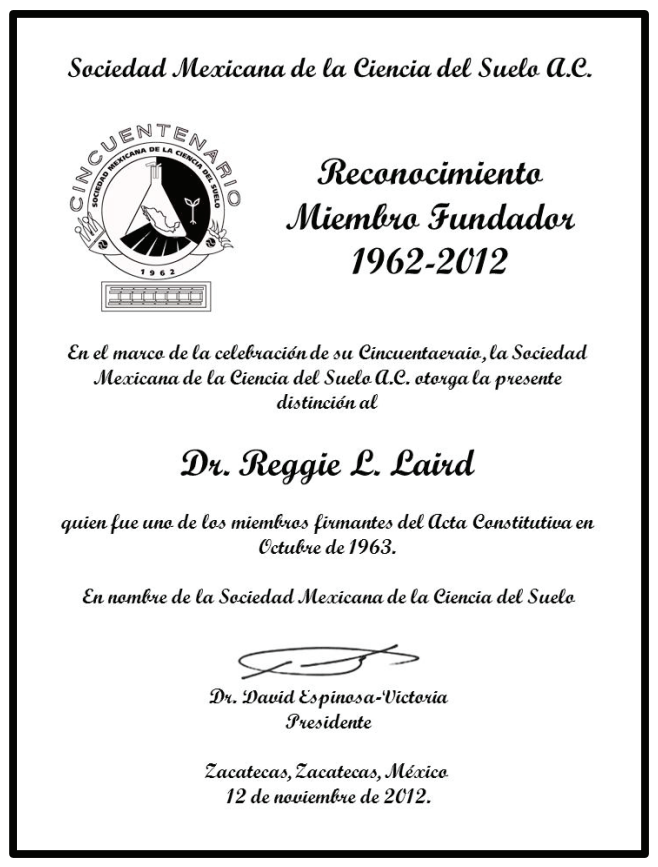

Figura 6. Reconocimiento otorgado en 2012 al Dr. Reggie J. Laird como miembro fundador de la Sociedad Mexicana de la Ciencia del Suelo, A.C., durante la celebración del Cincuentenario de la misma en el XXXVII Congreso Nacional en Zacatecas, Zac.

\section{Su Partida del Colegio de Postgraduados y del Mundo}

El Dr. Laird pasó 45 años de vida científica y docente en México. De estos, 38 años los dedicó como profesor investigador en el Colegio de Postgraduados. El Dr. Laird se retiró oficialmente del Colegio de Postgraduados el 1 de abril de 1997 (Colpos, 1999).

Su descenso ocurrió el 27 de febrero de 2014, a los 94 años de edad, en San Antonio, Texas, USA. Le sobreviven su esposa Rosario Laird, sus tres hijos Richard, David y Martha, así como 8 nietos. Finalmente, sus familiares y amigos le dedicaron el Servicio Memorial póstumo del 8 de marzo de 2014, en la Iglesia Presbiteriana de Álamo Heights de San Antonio, Texas, USA (Heidbrink, 2014).

\section{AGRADECIMIENTOS}

El autor expresa su agradecimiento al Dr. José I. Cortés Flores y al Dr. Antonio Turrent Fernández por la valiosa información y por sus atinados comentarios. 


\section{LITERATURA CITADA}

COLPOS. 1999. Colegio de Postgraduados. Semblanza sobre el Dr. Reggie J. Laird Broom. Doctorado Honoris causa. www. colpos.mx/honoris/rjlb.htm. (Consulta: septiembre 16, 2016).

Heidbrink, M. M. 2014. Laird's work with soil helped feed world. San Antonio Express-News. Friday, March 7.

Jiménez, S. L., J. I. Cortes F. y A. Turrent F. 2014. Reggie J. Laird 1920-2013. In Memoriam. 8. Fundación COLPOS.
Mendoza R., R., J. I. Cortés F., A. Turrent F., J. J. Castellón G. y J. Díaz A. 1994. Desarrollo de la multibarra portaimplementos de tracción animal línea Plan Puebla. Montecillo, Colegio de Postgraduados. ISBN: 9688391379.

Palacios R., M. I. 2011. Apuntes para la historia de la Sociedad Mexicana de la Ciencia del Suelo. pp. 47-94. In: M. I. Palacios rangel y J. A. Leos Rodríguez (eds.). La Sociedad Mexicana de la Ciencia del Suelo. Una historia de identidad compartida. Vol. 1. Universidad Autónoma Chapingo. ISBN: 978-607-120225-3 